\title{
Enquête épidémiologique préliminaire sur les prévalences des Salmonella spp. à l'abattoir de Bissau (Guinée-Bissau)
}

\author{
F.M.A. Bernardo ${ }^{1}$ C.F.S.N. Brandão ${ }^{1}$
}

\section{Mots-clés}

Bovin - Salmonella - Epidémiologie Abattoir - Prévalence - Sérotype Variation saisonnière - Antibiotique Guinée-Bissau.

\begin{abstract}
Résumé
Compte tenu de l'importance que revêtent les salmonelles comme agent de mortalité et de morbidité, notamment chez l'enfant, il a été décidé d'étudier la prévalence de ces bactéries avec la collaboration des autorités de GuinéeBissau, par une enquête épidémiologique à l'abattoir de Bissau. Le taux de prévalence sur 117 bovins abattus et reconnus aptes à la consommation humaine a été de 13,7 p. 100 , dont 8,5 p. 100 dans les prélèvements intestinaux et 5,1 p. 100 dans les vésicules biliaires. Les écouvillonnages rectaux, sur 74 autres animaux vivants, $n^{\prime}$ ont permis qu'un seul isolement : $S$. stanleyville. En revanche, aucune souche $n^{\prime}$ a été isolée dans les ganglions lymphatiques hépatiques. Ces souches, dont certains sérotypes sont pathogènes pour l'homme, sont en majorité dites "exotiques" : S. bargny, S. brazzaville, $S$. virchow, S. rubislaw, S. brazil, S. calabar, S. havana, S. hull, S. marseille, $S$. shipely, $S$. uppsala, $S .1 / 4,12 ; 1, w ; e, n, x$. Un nouveau sérotype est décrit : $S$. $28 ; f, m, t:-$. Isolé du contenu intestinal, ce sérotype présentait des fimbres MSHA $(++++)$ et une DL 50 de 7,0 Log 10. La majorité des souches présentaient une antibiorésistance, soit simple, soit multiple. Les isolements ont été plus fréquents en saison humide qu'en saison sèche.
\end{abstract}

\section{INTRODUCTION}

Les maladies diarrhéiques humaines sont responsables d'importants taux de mortalité et de morbidité $(15,21,28,30)$ dans les pays en voie de développement, surtout chez les enfants. L'importance nosologique des Salmonella qui sont des agents classiques de ces types de pathologies et leur présence chez les bovins abattus à Bissau et destinés à la consommation ont été mal évaluées (7, $10,14,20,23)$.

C'est pourquoi il est important de connaître la fréquence de portage sain des bovins destinés à la consommation humaine, dans la mesure où ils jouent, peut-être, un rôle d'agent opportuniste dans les cas de SIDA, ainsi, à litre d'exemple, que des schistosomoses. Le rôle des animaux domestiques et de ces produits comme réservoirs et véhicules de Salmonella est inconnu. La production et la consommation de viande de bovin sont très traditionnelles en Guinée-Bissau. C'est pourquoi il a été décidé d'effectuer une enquête épidémiologique chez les bovins abattus dans ce pays $(1,2$, $17,18,19,31)$ dont les résultats sont présentés ci-dessous.

\section{MATERIEL ET METHODES}

\section{Echantillonnage}

- Abattoir de Bissau : l'étude a été effectuée sur des bovins abattus acceptés pour la consommation humaine. La récolte des échan-

1. Faculdade de Medicina Veterinária, Rua Gomes Freire, 1199 Lisboa Codex, Portugal tillons a eu lieu pendant deux périodes distinctes : l'une en début de saison sèche, novembre et décembre 1990, l'autre en fin de saison sèche, février et mars 1991.

Pour chaque animal, il a été prélevé : le contenu caecal (CC), la vésicule biliaire (VB) et les ganglions lymphatiques hépatiques (GLH).

- Sur 74 animaux vivants (50 destinés à l'abattage et 25 vivant dans une exploitation), il a été pratiqué des écouvillonnages rectaux et pharyngiens. Tous les prélèvements ont été placés dans des flacons de Mackartney contenant $10 \mathrm{ml}$ d'eau peptonée tamponnée (Oxoid CM 509), maintenus réfrigćrćs et envoyés aussitôt que possible au Portugal pour analyse.

Il y a d'abord eu un préenrichissement en eau peptonée suivi d'un enrichissement en milieu sélénite-cystine (Difco 0687) et Rapapport-Vassiliadis (Oxoid CM 669) modifié avec 0,06 p. 100 d'agar (Difco 0140), dans des tubes en U (4).

\section{Isolement}

L'isolement a été effectué, à partir de chaque milieu d'enrichissement, sur deux géloses sélectives incubées à $37^{\circ} \mathrm{C}$ : vert brillant agar (Oxoid CM 329) et IIekthoen enteric agar (Difco 0853).

\section{Identification}

L'identification a été faite sur triple sugar iron agar (TSI, Difco 0265), complétée par le système API 20E (Bio-Mérieux 2010). 
Tableau I

Prévalence des bovins Salmonella-positifs en Guinée-Bissau

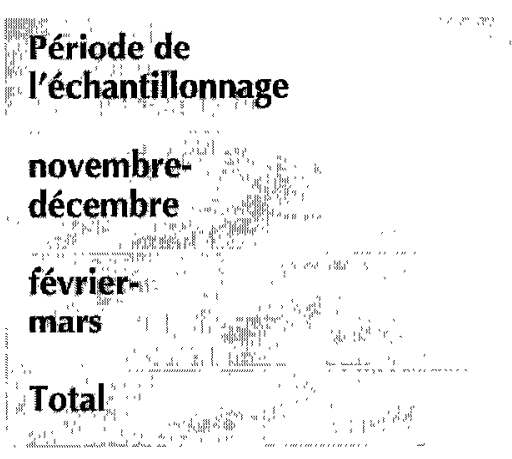

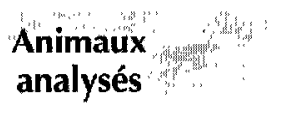

60

57

117

\section{CC}

$7(11,6 \%)$

$3(5,3 \%)$

$10(8,5 \%)$
Nature des prelevements
VB

$6(10 \%)$

0

$6(5,1 \%)$
Total

GLH

0

$13(21,6 \%)$

0

$3(5,3 \%)$

$0 \cdot 16(13,7 \%)$

$\mathrm{CC}=$ contenu caecal $; \mathrm{VB}=$ vésicule biliaire $; \mathrm{GLH}=$ ganglions lymphatiques hépatiques

\section{Sérotypage}

Il a d'abord été pratiqué avec des sérums anti-Salmonella polyvalcnts (Difco 2264). L'identification sérologique définitive a été effectuée au centre portugais de référence (Instituto nacional de Saúde Dr Ricardo Jorge), selon le schéma de Kaufmann-White.

\section{Antibiorésistance}

La sensibilité aux antibiotiques a été déterminée selon la méthode de Bauer et Kirby (3) en utilisant les disques antibiotiques suivants : ampicilline $10 \mu \mathrm{g}$ (Ap) (Difco 6363), chloramphénicol $30 \mu \mathrm{g}(\mathrm{Cm})$ (Difco 6113), gentamicine $10 \mu \mathrm{g}(\mathrm{Gm})$ (Difco 6423), céfotaxime $25 \mu \mathrm{g}(\mathrm{Clx})$ (Difco 6055), tétracycline $30 \mu \mathrm{g}$ (Tc) (Difco 6223), streptomycine $10 \mu \mathrm{g}$ (Sm) (Difco 6203), kanamycine $20 \mu \mathrm{g}(\mathrm{Km})$ (Difco 6263), nitrofurantoïne $300 \mu \mathrm{g}$ (Fd) (Difco 6713), acide nalidixique $30 \mu \mathrm{g}(\mathrm{Na})$ (Difco 6793) et sulfaméthoxazole + triméthoprime $25 \mu \mathrm{g}$ (SxT) (Difco 6831).

\section{RESUITATS}

\section{Efficacité des milieux de culture}

- Enrichissement : le milieu Rapapport-Vassiliadis montre une nette supériorité (100 p. 100 des souches isolées, suit 22 souches) par rapport au milieu sélenite - cystine (18 p. 100 des souches isolées, soit 4 souches).

- Isolement : le milieu de Hekthoen a montré une efficacité supérieure (72 p. 100 , soit 16 souches isolées) à celle du vert brillant $\operatorname{agar}(59,1$ p. 100 , soit 13 souches isolées).

La meilleure association de milieux enrichissement - isolement est la suivante : Rapapport - Vassiliadis + Hekthoen.

\section{Prévalence}

Les résultats sont donnés dans le tableau I. Pendant la période no vembre-décembre 1990, sur 60 animaux analysés, 13 étaient porteurs de salmonelles. La majorité des souches isolées $(62,5$ p. 100) provenaient du contenu caecal. Les restantes (37,5 p. 100) provenaient de la vésicule biliaire. Aucune souche n'a été isolée à partir des ganglions lymphatiques hépatiques. Une seule souche a été isolée pour 150 écouvillons rectaux, et aucune à partir des écouvillonnages pharyngiens.

\section{Sérotypes}

Les 22 souches isolées, toutes mobiles, se répartissent en 14 sérotypes, dont l'un est décrit pour la première fois : $S$. 28;f, $m, t$, : -
Parmi ces 22 souches, celle isolée à partir de l'écouvillon rectal est Salmonella stanleyville. Les résultats de ces sérotypes apparaissent dans le tableau II. Isolé du contenu intestinal, le sérotype $S$. 28; f, $m, t,:$ - présentait des fimbres MSHA (++++ pour une amplitude de - à ++++ chez les autres sérotypes) et une DL 50 de 7,0 Log 10 (pour une amplitude de 5,4 à 9,9 Log 10 chez les autres sérotypes).

\section{TABLEAU II}

Fréquences des sérotypes de Salmonella isolés de bovins en Guinée-Bissau

\begin{tabular}{|c|c|c|c|}
\hline Sérotypes & $\begin{array}{l}\text { Nombre } d^{\prime} \\
\text { isolements }\end{array}$ & $\begin{array}{l}\text { Fréquence } \\
(\text { p. 100) }\end{array}$ & Origine \\
\hline$\therefore$ & & & $\therefore$ \\
\hline S. brazzaville & 4 & 18,18 & VB \\
\hline S. virchow: & 4 & 18,18 & $\triangle B, C C$ \\
\hline S. bargny y & 3 & $13 ; 6$ & $\mathrm{CC}, \mathrm{VB}$ \\
\hline S. rubislaw & 1 & 4,5 & $\mathrm{CCH}_{3}$ \\
\hline S. brazil & 1 & 4,5 & $\mathrm{CC}$ \\
\hline S. calabar & 1 & 4,5 & $\mathrm{Ce}$ \\
\hline S. havana & 1 & 4,5 & $\mathrm{CC}$ \\
\hline S. hull & 1 & 4,5 & $\mathrm{CC}$ \\
\hline S. marscilld & 1 & $4,5: \cdots$ & $\mathrm{CC}$ \\
\hline S. shipely & 1 & 4,5 & $\mathrm{CC}$ \\
\hline S. uppsalla & 1 & 4,5 & VB \\
\hline S. 114,$12 ; 1, w ; e, n, x$ & 1 & 4,5 & $\mathrm{CC}$ \\
\hline S. $28 ; f, m, t:-$ & 1 & 4,5 & $\mathrm{CC}$ \\
\hline S. stanleyville & $1 \therefore \cdots$ & 4,5 & ER \\
\hline
\end{tabular}

$\mathrm{VB}=$ résicule biliaire $; \mathrm{CC}=$ contenu caecal $; \mathrm{ER}=$ écouvillon rectal

\section{Sensibilité aux antibiotiques}

La moitić des souches présente une résistance aux antibiotiques dont 45,5 p. 100 une résistance simple, et 54,5 p. 100 une résistance multiple. Les résultats sont exprimés dans les tableaux III et IV.

\section{DISCUSSION}

Le portage de salmonelles semble subir une variation très marquée en fonction des saisons. Il semble en liaison avec des variations climatiques, notamment d'humidité relative. En fin de saison des pluies et début de saison sèche, 21,6 p. 100 des animaux étaient positifs, alors qu'en fin de saison sèche seulement 5,3 p. 100 

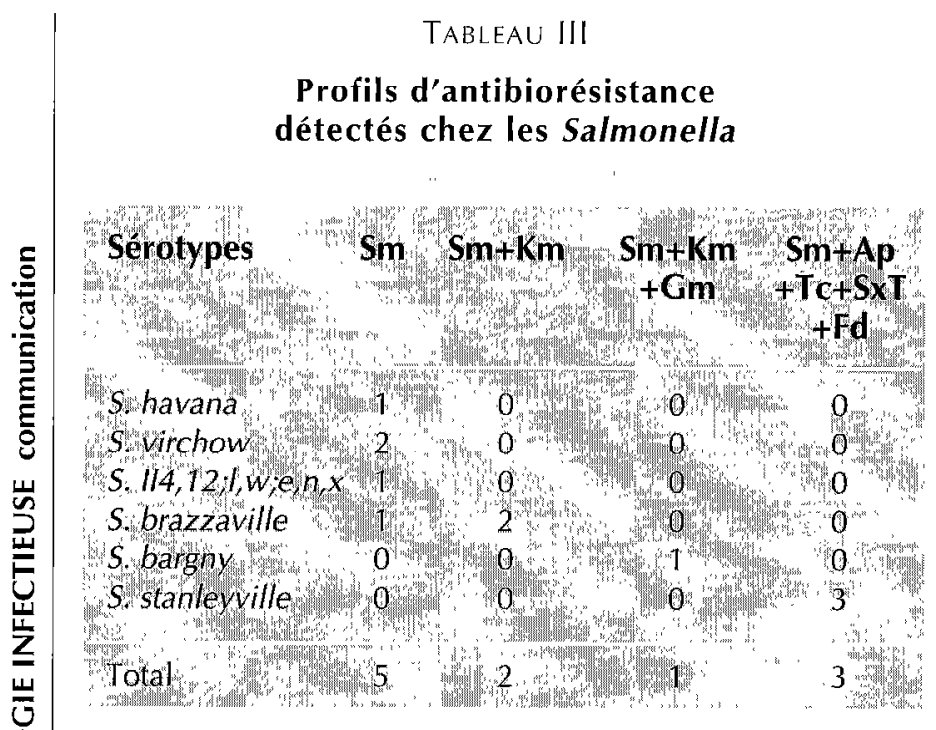

$\mathrm{Sm}=$ streptomycine $; \mathrm{Km}=$ kanamycine $; \mathrm{Gm}=$ gentamicine $; \mathrm{Ap}=$ ampicilline $;$ $\mathrm{Tc}=$ tétracycline $; \mathrm{Fd}=$ nitrofurantö̈ne $; \mathrm{SxT}=$ sulfamétoxazole+triméthoprime

TABLEAU IV

\section{Fréquence de la résistance aux différents antibiotiques}

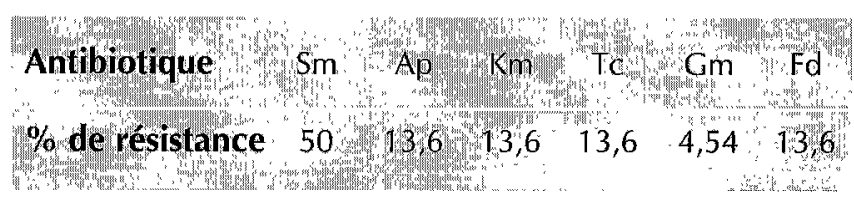

l'étaient. Il semble y avoir une concordance avec des cas de pathologie humaine en Afrique, puisqu'à l'hôpital de Libreville, de juillet 1981 à juin 1983, une prévalence plus élevée a été observée chez des enfants en saison des pluies (23).

Les résultats observés lors de cette enquête sont comparables à ceux obtenus lors d'enquêtes au Niger (32) ou en Arabie Saoudite (28) bien que ces derniers résultats concernaient des ovins. Lors d'une autre enquête, au Nigeria, on a trouvé des résultats inférieurs (1), mais la méthodologie utilisée était vraisemblablement différente, d'une part par le nombre d'échantillons examinés, et d'autre part par la recherche de salmonelles en plusieurs points des carcasses $(16,26,29)$. En fonction de la nature des échantillons, les rapports de pourcentage des isolements peuvent aussi évoluer $(5,25)$. Dans le même ordre d'idées, pour des raisons techniques, il n'a pas été possible de faire des prélèvements au niveau du matériel et des installations de l'abattoir, ce qui aurait permis d'avoir des données plus précises sur la localisation des germes $(1,6)$.

Les sérotypes isolés lors de cette enquête sont trouvés régulièrement dans d'autres Etats africains, sur différentes espèces animales, y compris l'homme. C'est le cas de $S$. bargny $(6,9,11,12$, $27,33)$, de $S$. rubislaw $(7,11,27,33)$, de $S$. havana $(6,7,8,9,11$, $12,22,25)$, de $S$. hull $(6,11,27,33)$, de $S$. marseille $(11)$, de $S$. stanleyville $(1,13,28,33)$, et de $S$. uppsalla (5). S. stanleyville paraît fréquemment isolée en Afrique centrale.

\section{- CONCLUSION}

Malgré la petite taille de l'échantillonnage prélevé, il est possible de tirer quelques conclusions concernant la prévalence des salmo- nelles sur des bovins abattus et reconnus propres à la consommation humaine. Les résultats obtenus sont sensiblement analogues à ceux relatés par les pays voisins. Les conditions sanitaires propres à l'abattoir de Bissau peuvent être des causes de dispersion des germes et de contamination des viandes et de l'environnement, mais ce facteur n'a pas pu être étudié en détail et mériterait une étude approfondie avec pour finalité la mise en place d'un système HACCP (hazard analysing critical control points).

Dans cette enquête, des souches pouvant infecter 1'homme ont été isolées ( $S$. bargny, S. rubislaw, S. havana, S. hull, S. marseille, $S$. stanleyville), ce qui pose des problèmes notamment dans les diarrhées chez l'enfant, compte tenu du fait que l'antibiorésistance est fréquente.

\section{Remerciements}

Nous remercions tout le personnel du laboratoire de la Direction générale de Pécuaire, de l'Abattoir et de la Mairie de Bissau et, au Portugal, de l'Institut national de santé Dr Ricardo Jorge, du Laboratoire national d'investigation vétérinaire, du Laboratoire d'inspection sanitaire de la Faculté de médecine vétérinaire et du Centre vétérinaire et de zootechnie, M. Ch. Meyer du CIRADEMVT, France, pour son aide dans la rédaction finale de l'article en français, et toutes les autres personnes qui nous ont aidés.

\section{BIBLIOGRAPHIE}

1. ADESIYUN A.A., ONI O.O., 1989. Prevalence and antibiograms of Salmonella in slaugther cattle, slaughter areas and effluents in 7 aria abattoir, Nigeria. J. Food. Protec., 52: 232-235.

2. A7EVEDO J.F., XAVIER M.L.S., 1965. Um novo vector potencial do Shistosoma haematobium em Portugal. Anais Inst. Med. trop., 22 : $35-$ 47.

3. BAUER A.W., KIRBY, SHERRIS J., TURCK M., 1966. Antibiotic susceptibility testing by standardised single disk method. Am. J. clin. Pathol., 45: 493-496.

4. BERNARDO' F.M.A., 1991. Significado epidemiológico da incidência de Salmonella em alguns alimentos de origem animal em Portugal. Tese Doutoramento,' Faculd. Med. vet. Univ. tec. Lisboa, Portugal.

5. CHAMBERS P.G., 1991. Salmonella in Rhodesia; sources and serotypes of same isolates from abattoirs, domestic animals, birds and man. J. South Afr. vet. Assoc., 48: 241-243.

6. CHAMBRON J., DOUTRE M.P., SARRAT H., MARTEL J.L. 1971. LeS salmonelloses au Sénégal. Importance des rapaces anthropophiles de la région du Cap Vert en tant que réservoir de salmonelles. Revue Elev. Méd. vét. Pays trop., 24 : 9-18.

7. CHAMBRON J., MARTEL J.L., SARRAT H., DOUTRE M.P., 1971 Isolement de 28 souches de Salmonella à partir de ganglions mesentériques des porcs sains abattus à Dakar. Revue Elev. Méd. vét. Pays trop., 24 : 497-504.

8. DOUTRE M.P., BOCHE R., 1976. Sérotypes de Salmonella isolés chez les petits ruminants abattus à Dakar. Revue Elev. Méd. vét. Pays trop., 29 : 205-209.

9. DOUTRE M.P., CARTEL J.L., 1979. Sérotypes de Salmonella isolés chez les bovins et les chevaux du Sénégal. Revue Elev. Méd. vét. Pays trop., 32 : 19-23.

10. DOUTRE M.P., CHAMBRON J., SAGNA F., SARRAT H., BOCHE R., CARTEL J.L., 1980. Les salmonelloses animales au Sénégal. Ann. Inst. Pasteur, 131 : 105. 
11. DOUTRE M.P., BUISSON Y., 1984. Sérotypes de Salmonella isolés chez I'animal au Sénégal. Revue Elev. Méd. vét. Pays trop., 37 : 123128.

12. DOUTRE M.P., SARRAT H., 1973. Sérotypes de salmonelles isolés chez les chiroptères frugivores et insectivores du Sénégal - Importance épidémiologique. Revue Elev. Méd. vét. Pays trop., 26 : 279-287.

13. DUBE S.D., BHAGWAT A.G., 1983. Non-typhoidal Salmonella infection in Zambian infants. Trans. R. Soc. trop. Med. Hyg., 77: 336-337.

14. GENDREL D., SITBON M., LENOBLE D.R., GALLIOT A., KOMBILA M., NARDOU M., GENDREL G., KANI F., 1985. Etiologies des gastroentérites aiguës infantiles au Gabon. Bull. Soc. Path. exot., 78 : 290-295.

15. GRACEY M., IVLSON J.B., SUNOTO SUHARYONO., 1980. Human Salmonella carriers in a tropical urban environment. Trans. R. Soc. trop. Med. Hyg., 74: 479-482.

16. GUINE P.A.M., KAMPELMACHER E.H., VANKEULEN A., HOFSTRA K., 1964. Salmonella in healthy cows and calves in Netherlands. 7 bl. Bkt. B. 11: $728-740$.

17. HALAWANI A., ABDALLA A., BADRAN A., 1960. The relation bctween schistosomiasis and the urinary Salmonella carrier state. Am. J. trop. Med. Hyg., 9: 371-373.

18. HATOUT S.E.D., GHAFFAR Y.A.E., AWNY A.Y., 1967. Salmonellosis complicating schistosomiasis in Egypt. Am. J. trop. Med. Hyg., 16: 462-472.

19. LE MINOR L., 1994. Salmonella, Lignières 1990. Bergey's Manual of systematic bacteriology, Vol. 1, 9th ed. Baltimore, USA, Williams \& Wilkins.

20. MAFAMA N.K., MANYA T., KALOMBO M., 1982. Epidémiologie des salmonelloses chez quelques espèces animales au Zaïre. Revue Flev. Méd. vét. Pays trop., 35 : 221-224.

21. MATA L., SIMIION A., PADILLA R., GAMBO^ M.M., VARGAS G., HERNANDEZ F., MOHS E., LIZANO C., 1983. Diarrhea associated with rotaviruses enterotoxigenic Escherichia coli, Campylobacter, and other agents in Costa Rican children, 1976-1981. Am. J. trop. Med. Hyg., 32 : 146-153.

22. M'BOUP S., DAVID M.P., DENIS F., SY I., CORREA P., 1980. Epidémie de diarrhées aiguës mortelles du nouveau-né due à une souche de Salmonella multirésistante : S. mbao. Ann. Inst. Pasteur, 131A : 103-104.
23. MEFAME C., OMWANGA D., 1986. Sérologie, sensibilité aux antibiotiques et lysotypie de quelques souches de Salmonella isolées chez les enfants hospitalisés au centre hospitalier de Libreville. Bull. Soc. Path. Exot., $7:$ 165-171.

24. MENDES M., 1971. Algumas doenças dos animais em Angola e Moçambique e sua importância na higiene das carnes. Rev. Port. Ciênc. Veter, $66: 271-286$.

25. NABBUT N.H., AL-NAKHLI E.H.M., 1982. Incidence of Salmonella in lymphnodes, spleens and feces of sheep and goats slaughtered in the Riyadh public abattoir. J. Food Protec., 45: 1314-1317.

26. NAZER N.H.K., OSBORNE A.D., 1976. Salmonella infection and contamination of veal calves: a slaughterhouse survey. $\mathrm{Br}$. vet. J., 132: 617-622.

27. PERPEZAT A., PERREAU P., THOME M., Vicilek M., 1964. Différents sérotypes de Salmonella isolés en République du Tchad. Revuc Elcv. Mćd. vćt. Pays trop., 17 : 35-11.

28. PETAT E., CARTERON B., REGUER M., LEMMENS P., VANDEPITTE J., GHYSELS G., 1987. Etude des Shigella et Salmonella isolées au Burundi de 1980 à 1985. Bull. Soc. Path. exot,, 80 : 171-176.

29. SAMUEL J.L., O'BOYLE D.A., MATHERS W.J., FROST A.J., 1980. Distribution of Salmonella in the carcasses of normal cattle at slaughter. Res. vet. Sci., 28: 368-372.

30. SHETTY N., NARASHIMA M., RAGHUVEER T.S., ELLIOT E., FARTHING M.J.G., MACADEN R., 1990. Intestinal amebiasis and giardisis in Southern Indian infants and children. Trans. R. Soc. trop. Med. Hyg., 84: 382-384.

31. TAUXE R.V., 1991. Salmonella: A postmodern pathogen. J. Food Prot., 54 (7): 563-568.

32. UZOUKWU M., MOIIAN K., 1990. Intestinal pathology associated with Salmonella in apparently healthy animals. Bull. Anim. Health Prod. Afr., 38: 351-353.

33. VIGIER M., CHAMOISEAU G., 1967. Différents sérotypes de Salmonella isolés au Tchad. Revue Elev. Méd. vét. Pays trop., 20 : 61-65.

Reçu le 8.3.94, accepté le 2.8.96 


\section{Summary}

Bernardo F.M.A., Brandão C.F.S.N. Preliminary survey on Salmonella prevalence at Bissau slaughterhouse (GuineaBissau)

Considering the importance of Salmonclla as a mortality and morbidity agent, in particular in children, a study on Salmonella prevalence was performed in collaboration with the Guinea-Bissau authorities through an epidemiological survey at Bissau slaughterhouse. The prevalence rate in 117 bovines slaughtered and approved for human consumption was $13.7 \%$, with $8.5 \%$ sampled in the intestine and $5.1 \%$ in the gallbladder. Rectal swabbing in 74 live animals resulted in the isolation of $S$. stanleyville only. On the other hand, no strain was found in hepalic lymphnodes. These strains, some of them pathogenic for humans, are mostly called "exotic": $S$. bargny, S. brazzaville, S. virchow, S. rubislaw, S. brazil, $S$. calabar, S. havana, S. hull, S. marseille, S. shipely, S. uppsala, S. $1 / 4,12 \%, w ; e, n, x$. A new serotype is described: $S$. 28,f, $m, t:-$. Isolated from the caecal content, it had MSHA fimbriae $(++++)$ and $7.0 \log 10$ as DL 50 . Most of these strains presented simple or multiple antibiotic resistance. They were more frequently isolated during the wet season than during the dry season.

Key words: Bovine - Salmonella - Epidemiology - Abattoir Prevalence - Serotype -Seasonal variation - Antibiotics Guinea-Bissau.

\section{Resumen}

Bernardo F.M.A., Brandão C.F.S.N. Encuesta epidemiológica preliminar sobre las prevalencias de salmonella spp en el matadero de Bissau (Guinea-Bissau).

Dada la importancia que prescntan las salmonclas como agentes de mortalidad y de morbilidad, principalmente en el niño, se decidió estudiar la prevalencia de estas bacterias, en colaboración con las autoridades de Guinea Bissau y mediante una encuesta epidemiológica en el matadero de Bissau. La tasa de prevalencia sobre 117 bovinos sacrificados y marcados aptos para el consumo humano, fue de 13,7 por ciento, de los cuáles 8,5 por ciento en muestras intestinales y 5,1 por ciento en las vesículas biliares. Las muestras rectales obtenidas sobre olrus 74 animales vivos, permitieron únicamente un aislamiento: $S$. stanleyville. Por otro lado, ninguna cepa fue aislada en ganglios linfáticos hepáticos. Estas cepas, de las cuáles algunos serotipos son patógenos al hombre, son generalmente llamadas "exóticas": S. bargny, S. brazzaville, S. virchow, S. rubislaw, S. brazil, S. calabar, S. havana, S. hull, S. marseille, S. shipely, S. uppsala, S. $114 ; 1 ; w ; e ; n ; x ;$ Se describe un nuevo serotipo: $S .28, t, m, t ;--$. Este serotipo aislado, del contenido intestinal, presentó fimbrias MSHA (++++) y una DL50 de 7,0 Log 10. La mayoría de las cepas presentaron una resistencia a los antibióticos, ya sea simple o múltiple. Los aislamientos fueron más frecuentes durante la estación húmeda que diurante la seca.

Palabras clave : Ganado bovino - Salmonella - Epidemiología - Matadero - Prevalencia - Serotipo - Variación estacional Antibiótico - Ciuinea-Bissau. 